Nutzen für Brustkrebspatientinnen mit ZNS-Metastasen

Patientinnen mit HER2-positivem metastasiertem Mammakarzinom profitieren von der Zweitlinentherapie mit Trastuzumab Emtansin (Kadcyla ${ }^{\oplus}$ ). Das Antikörper-Wirkstoff-Konjugat verlängert das Gesamtüberleben (OS) der betroffenen Frauen gegenüber Capecitabin/Lapatinib sowie HER2-basierten Vergleichstherapien um etwa ein halbes Jahr. Eine aktuelle Interimsanalyse der Sicherheitsstudie KAMILLA, die auf dem San Antonio Breast Cancer Symposium (SABCS) 2016 vorgestellt wurde, zeigt, dass auch Frauen mit Metastasen im zentralen Nervensystem (ZNS) von der Behandlung profitieren können [Montemurro $\mathrm{F}$ et al. SABCS. 2016;Poster P1-12-10]. Bei zwei von drei Frauen mit messbaren ZNSMetastasen zu Studienbeginn verkleinerten sich die Läsionen im Gehirn durch die Behandlung. Darüber hinaus erreichten Frauen mit ZNS-Metastasen zu Studienbeginn ein medianes progressionsfreies Überleben (PFS) von 5,5 Monaten.

Nach Information von Roche

\section{Zusatznutzen für Nivolumab bei RCC bestätigt}

Der Gemeinsame Bundesausschuss (G-BA) hat seinen Beschluss über Nivolumab $\left(\mathrm{Opdivo}^{\oplus}\right.$ ) als Monotherapie bei Erwachsenen zur Behandlung des fortgeschrittenen Nierenzellkarzinoms (RCC) nach Vortherapie veröffentlicht. Für Patienten nach antiangiogener Vortherapie erkennt der G-BA einen Hinweis auf einen beträchtlichen Zusatznutzen. Für Patienten, die mit Temsirolimus vorbehandelt sind, wurde der Zusatznutzen nicht bestätigt. Die Bewertung des Zusatznutzens für Patienten nach antiangiogener Vortherapie basiert auf den Ergebnissen der Phase-III-Studie CheckMate-025. In dieser wurde Nivolumab gegenüber Everolimus bei Erwachsenen mit RCC nach Vortherapie mit maximal zwei Angiogenesehemmern untersucht. Nach einer Interimsanalyse wurde die Studie vorzeitig beendet, weil sich ein deutlicher Vorteil beim Endpunkt Gesamtüberleben (OS) abzeichnete. Mit Nivolumab behandelte Patienten lebten im Median deutlich länger ( 25,0 vs. 19,6 Monate unter Everolimus; Hazard Ratio 0,73, 98,5\%-Konfidenzintervall $0,57-0,93 ; p=0,0018$ ).

Nach Information von Bristol-Myers Squibb

\title{
Pankreaskarzinom: Neue Zulassung für die Zweitlinientherapie
}

\begin{abstract}
— Für Patienten mit metastasiertem Pankreaskarzinom steht mit der ersten liposomalen Formulierung von Irinotecan (nal-IRI) ab sofort ein neuer Wirkstoff zur Verfügung, der in der Zweitlinientherapie nach Gemcitabin zum Einsatz kommen kann. Die Zulassung mit Orphan-Drug-Status basiert auf der Phase-III-Studie NAPOLI-1, in der nal-IRI (Onivyde ${ }^{\circledR}$ ) in Kombination mit 5-FU/LV (5-Fluorouracil/Folinsäure) gegenüber 5-FU/ LV allein bei allen Wirksamkeitsparametern besser abschnitt. An der Studie hatten Patienten mit metastasiertem Pankreaskarzinom und Progress nach einer Gemcitabin-basierten Therapie teilgenommen. Unter der Kombination mit nal-IRI lag das mediane Gesamtüberleben bei 6,1 Monaten, gegenüber 4,2 Monaten unter 5-FU/LV allein $(p=0,012$ ) [Wang-Gillam A et al. Lancet. 2016;387(10018):545-57]. Das progressionsfreie Überleben (PFS) betrug 3,1 versus 1,5 Monate $(p=0,0001)$, beim objektiven und
\end{abstract}

beim biochemischen Ansprechen zeigte sich ebenfalls eine signifikante Überlegenheit ( $p<0,0001$ bzw. $p=0,0006)$. Unter der nal-IRI-Kombination hätte es zwar mehr Nebenwirkungen gegeben, doch diese seien für den Patienten oft nicht bemerkbar und gut behandelbar gewesen, erläuterte Arndt Vogel, Hannover. Er geht davon aus, dass nal-IRI plus 5-FU/LV der Standard in der Zweitlinientherapie nach Gemcitabin werden wird. Die NAPOLI-Studie biete die beste Evidenz zur Zweitlinientherapie und die Verträglichkeit sei vergleichsweise gut. Gemäß Vogel wird nal-IRI plus 5-FU/LV derzeit mit bzw. ohne Oxaliplatin in einer Phase-II-Studie auch in der Erstlinientherapie des metastasierten Pankreaskarzinoms untersucht.

Petra Eiden

Launch-Pressekonferenz "Onivyde ${ }^{\circledR}$ - Neue Perspektive für Patienten mit fortgeschrittenem Pankreaskarzinom", Berlin, 18. November 2016; Veranstalter: Shire

\section{Kombinationstherapie bei fortgeschrittenen Weichgewebesarkomen zugelassen}

— Der humane, monoklonale Antikörper Olaratumab wurde im November 2016 von der europäischen Arzneimittelbehörde (EMA) in Kombination mit Doxorubicin zur Behandlung von Patienten mit fortgeschrittenem Weichgewebesarkom zugelassen. Eingesetzt werden kann Olaratumab (Lartruvo $\left.{ }^{T M}\right)$, wenn die Betroffenen nicht für eine kurative Therapie geeignet sind, und wenn sie zuvor nicht mit Doxorubicin behandelt wurden.

"Mit dieser Zulassung, die von der europäischen Arzneimittelbehörde EMA beschleunigt erteilt wurde, steht für Patienten mit fortgeschrittenen Weichgewebesarkomen erstmals seit 40 Jahren eine neue Erstlinientherapie zur Verfügung", berichtete Peter Reichardt, Berlin-Buch.

Basis der Zulassung waren die Ergebnisse der offenen und randomisierten Phase-IIStudie JGDG, in der Olaratumab in Kombination mit Doxorubicin mit einer alleinigen Doxorubicin-Gabe bei nicht kurativ therapierbaren Patienten mit fortgeschrittenem
Weichgewebesarkom verglichen wurde [Tap WD et al. Lancet. 2016;388(10043):48897]. Unter der Kombinationstherapie erreichten die Patienten im Vergleich zu Doxorubicin allein ein medianes progressionsfreies Überleben von 6,6 versus 4,1 Monaten (Hazard Ratio [HR] 0,672; $95 \%$-Konfidenzintervall [95\%-KI] 0,442-1,021; $\mathrm{p}=0,0615$ ) sowie ein statistisch signifikant längeres Gesamtüberleben von 26,5 versus 14,7 Monaten (HR 0,463, $95 \%-K I$ 0,301-0,701; $p=0,0003)$.

„Damit lebten die Patienten unter der Olaratumab/Doxorubicin-Kombination im Median 11,8 Monate länger als unter Doxorubicin allein. Das Sterblichkeitsrisiko hat sich mehr als halbiert - ein sensationell gutes Ergebnis für diese schwer zu behandelnden, seltenen Tumoren", kommentierte Reichardt die Daten.

Silke Wedekind

Launch-Pressekonferenz „Im Fokus: Olaratumab erhält beschleunigte EU-Zulassung beim fortgeschrittenen Weichgewebesarkom", Berlin, 18. November 2016; Veranstalter: Lilly 